\title{
Management of chronic scrotal content pain
}

\author{
Christopher Wu, MD, FRCSC; Keith Jarvi, MD, FRCSC
}

Division of Urology, University of Toronto, Toronto, ON

Cite as: Can Urol Assoc J 2018;12 (6Suppl3):S164-6. http://dx.doi.org/10.5489/cuaj.5326

\section{Introduction}

Chronic scrotal content pain (CSP) is a common, yet poorly understood condition that significantly impacts quality of life. This condition is defined as an intermittent or constant, unilateral or bilateral pain localized to the scrotal structures, three months or longer in duration that significantly interferes with daily activities of the patient and prompts him to seek medical attention. Though the true incidence of the condition in the population is not known, several studies have shown that up to $4 \%$ of men presenting for urologic assessment will identify as having CSP.

\section{Practice tips}

\section{Tip 1: History and physical are paramount}

These patients should be carefully evaluated for potentially reversible causes of scrotal pain. From our experience, the most common identifiable causes of pain are prior vasectomy, varicoceles, inguinal hernia repairs, cystic scrotal conditions (such as spermatoceles or hydroceles), and infections of the epididymis/testis. Many of these patients may also have coexisting chronic prostatitis/chronic pelvic pain syndrome (CP/CPPS). Some of these patients may also present with referred pain from nearby structures. A significant proportion of patients assessed in our CSP clinic had pain in the adductor tendons, hernias, or pain at the insertion of the inguinal ligament, suggesting a musculoskeletal cause. Recognizing these conditions through thorough patient evaluation can help direct management.

\section{Tip 2: Manage expectations from the initial visit}

As with many chronic pain syndromes, patients are usually quite frustrated with their condition and many may have seen multiple healthcare providers and tried different treatment modalities with no significant benefit. It is important to educate patients that the natural history of the condition is poorly understood. Though many treatments show promise,

their pain may not completely resolve. In certain patients, unilateral CSP may switch to the other side after treatment of the initially affected side. There are also a number of individuals in whom the pain resolves spontaneously without intervention. Up to $50 \%$ of patients will not have an identifiable etiology of pain.

\section{Tip 3: Consider the psychosocial implications of their pain}

It is important to assess the impact of CSP on the patient's life. Many of these patients may exhibit symptoms of depression and can become socially isolated. Many patients may develop pain-distorted thinking, which impacts social/occupational functioning, leading to maladaptive self-harming behaviours and feelings of helplessness. If significant psychological distress is recognized, referral a mental health specialist is advised. Psychotherapy, such as cognitive behavioural therapy, has shown benefit in other in chronic pain syndromes, such as CP/CPPS, to increase activity and reduce pain-related limitations.

Tip 4: Scrotal ultrasound can be a useful tool, but not all findings are clinically significant

Although the evidence for ultrasound assessment in the CSP patient with a completely normal physical examination is limited, it can be a useful diagnostic measure for patients with abnormalities on examination, or in those whose pain or habitus precludes a proper examination. In addition to this, many patients and clinicians may be reassured that there is no contributing pathology when an ultrasound is obtained. It is important to exercise common sense when interpreting imaging results, as certain imaging findings may not necessarily be the cause of pain, such as sublinical epididymal cysts or many varicoceles, especially since these are present in many patients without CSP. If any unexpected findings are present, it is important to clinically correlate this in order to avoid unnecessary procedures that will not benefit the patient.

\section{Tip 5: Neuropathic medications can be a useful adjunct to therapy}

Many patients with CSP have a neuropathic pain component. Neuropathic medications, such as nortriptyline or gabapentin/pregabalin, may effectively control pain, especially when 
used in conjunction with anti-inflammatory medications. In this patient population, the use of neuropathic medications may help spare the use of opioid medications, which carry significant addictive potential, especially when used for chronic pain.

\section{Tip 6: Physical therapy should be considered in certain patients}

Up to $10 \%$ of patients presenting with CSP are found to have musculoskeletal pain localizing to areas such as the conjoint tendon, the adductor tendon, or the pelvic floor. These patients may benefit from physical therapies, such as myofascial trigger point release, perineal/pelvic floor massage, or other targeted physiotherapy to these areas.

\section{Tip 7: Vasovasostomy is effective for post-vasectomy pain}

Although the current evidence for management of post-vasectomy pain syndrome (PVPS) is limited, multiple single-centre studies have shown that nearly $100 \%$ of patients will have improvement in pain scores with microsurgical vasovasostomy for pain. Patients presenting with CSP and a history of prior vasectomy should be referred to a microsurgery facile urologist for consideration of a microsurgical vasovasostomy.

Tip 8: Epididymectomy may be effective for patients with symptomatic epididymal cysts

Although the success rates for epididymectomy for pain vary widely (from 10 to $>90 \%$ ), studies have shown that in patients with scrotal pain localized to palpable epididmyal pathology, such as painful cysts, had much higher success rates with epididymectomy. Pain resolution rates in these specially selected patients approached $75 \%$.

Tip 9: Nerve blocks are usually of short duration and of limited clinical utility, but identify men who are candidates for spermatic cord denervation

Spermatic cord blocks may provide temporary relief of the scrotal pain, but for most men, the pain recurs in hours to days. The block is, however, useful to predict the success of the spermatic cord denervation procedure. It is possible that repetitive cord blocks could reduce pain through a neural down regulation process and is worth considering in selected patients who respond well with initial injection.

Tip 10: Microsurgical denervation of the spermatic cord is an emerging treatment for CSP

Microsurgical denervation of the spermatic cord (MDSC) was first introduced in 1978 to treat testicular pain of unknown etiology, and this procedure has steadily increased in popu- larity over the past decade, with better understanding of the pathophysiology of CSP. Through the transection of all the cord structures except for the testicular artery and lymphatics, the different nerve plexuses running through the spermatic cord are ablated. Multiple retrospective studies looking at the efficacy of MDSC have reported success rates ranging from $71-96 \%$. A positive response to spermatic cord blockade can predict resolution of pain with MDSC.

This is a procedure with significant complications, including testicular atrophy from vascular injury, and thus, should be performed in dedicated centres with microsurgical capability and expertise in the MDSC technique.

\section{Tip 11: If orchiectomy is being considered, an inguinal approach should be used}

An orchiectomy remains a surgical option, especially for those with significant ongoing pain in which all other options have failed. A scrotal approach should be avoided, as an inguinal/ sub-inguinal approach appears to result in superior success rates. This may be reflected in the ability to achieve a higher ligation of the ilioinguinal or genitofemoral nerve with this approach, addressing any neuropathic components of pain.

\section{Tip 12: Treatment is often multidisciplinary}

As urologists, we are experts in the area of urology. Referring patients for multidisciplinary and often multimodal care at tertiary care centres or at specialized pain clinics allows urologists to continue to work in their "comfort zone" of urology, while offering the patients the full care needed.

\section{Summary}

CSP is a common, complex, yet poorly understood condition that can be frustrating for both patients and urologists. Thorough evaluation of the patient is important, and it is important to recognize the psychosocial implications of pain. Multimodal treatment approaches using lifestyle measures, physical therapy, and psychotherapy may empower patients and provide useful tools for coping with this condition. Neuropathic medications are promising alternatives to opioid analgesics for chronic pain conditions. There are promising surgical options emerging for CSP. An algorithm of conservative and medical management of idiopathic CSP is shown in Fig. 1.

Competing interests: Dr. Jarvi has participated in clinical trials supported by Allergan. Dr. Wu reports no competing personal or financial conflicts related to this work.

This paper has been peer reviewed. 
Wu \& Jarvi

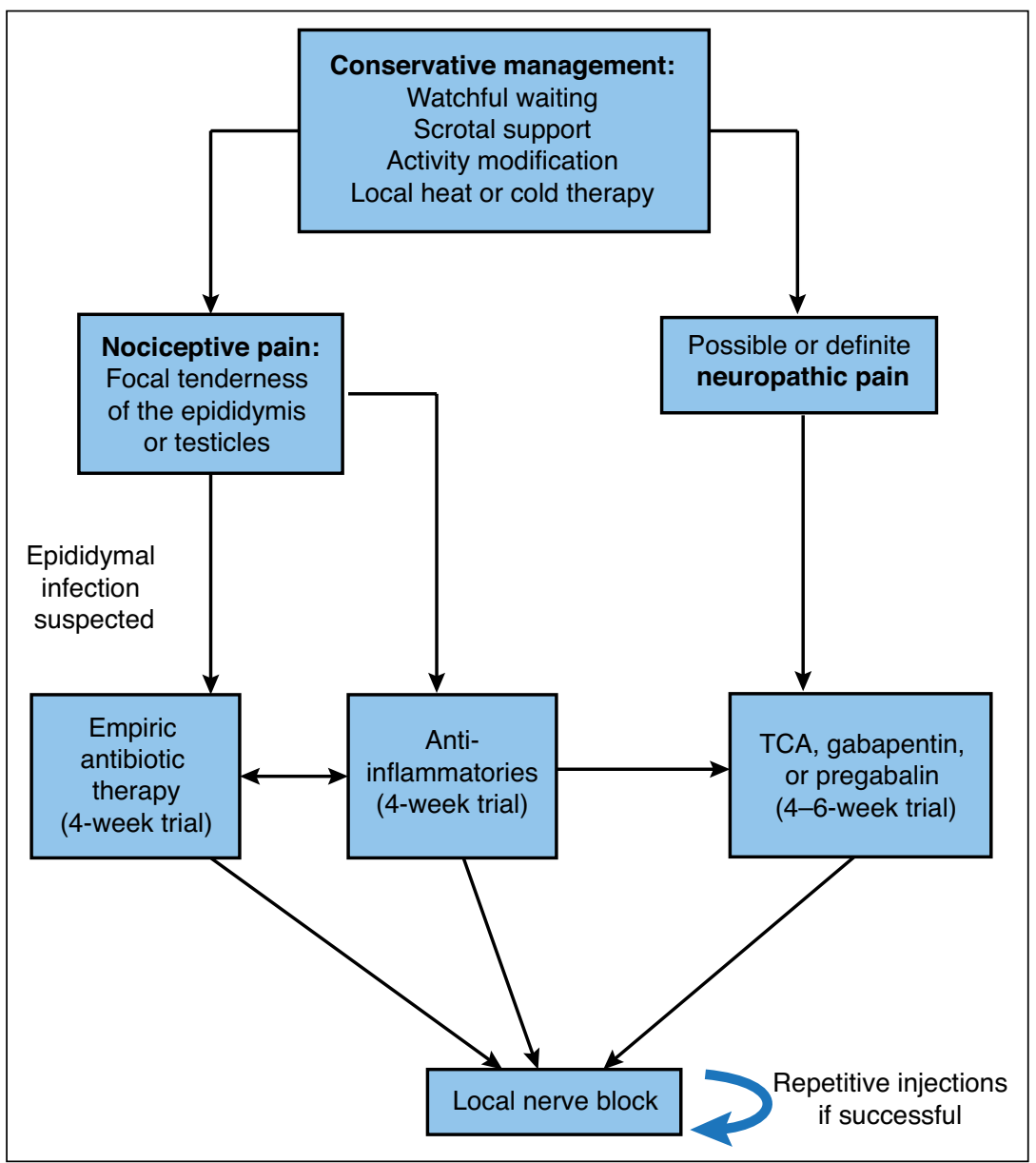

Fig. 1. Conservative and medical management of idiopathic chronic scrotal pain. TCA: tricyclic antidepressant.

\section{Recommended reading}

1. Lian F, Shah A, Mueller B, et al. Psychological perspectives in the patient with chronic orchialgia. Iransl Androl Urol 2017;6:S14-9. https://doi.org/10.21037/tau.2017.03.91

2. Davis $B E$, Noble MJ, Weigel JW, et al. Analysis and management of chronic testicular pain. J Urol 1990;143:936-9. https://doi.org/10.1016/S0022-5347(17)40143-1

3. Masarani $M$, Cox R. The etiology, pathophysiology, and management of chronic orchialgia. BJU Int 2003;91:435-7. https://doi.org/10.1046/i.1464-410X.2003.04094.
4. Jarvi KA, Wu C, Nickel JC, et al. A Canadian Urological Association best practice report on chronic scrotal pain. Can Urol Assoc J 2018 Feb. 23; Epub ahead of print. http://dx.doi.org/10.5489/cuaj.5238

Correspondence: Dr. Keith Jarvi, Division of Urology, University of Toronto, Toronto, ON; Keith.Jarvi@sinaihealthsystem.ca 